\title{
Research of the Sensitivity of the Quantized Coefficients of a Digital Bandpass Filters with Frequency Sampling
}

\author{
Rybka Serhii Volodymyrovych ${ }^{1}$, Korolov Andrii Pavlovych ${ }^{2}$, Varava Ivan Andriiovych ${ }^{3}$ \\ ${ }^{1}$ Pukhov Institute for Modelling in Energy Engineering National Academy of Sciences of Ukraine, \\ ${ }^{2}$ Odessa National O.S. Popov Academy of Telecommunications, Ukraine \\ ${ }^{3}$ Igor Sikorsky Kyiv Polytechnic Institute, National Technical University of Ukraine
}

\begin{tabular}{|c|c|}
\hline Article Info & ABSTRACT \\
\hline Article history: & $\begin{array}{l}\text { The article is devoted to the applied research of the dependence of the } \\
\text { influence of the transfer function quantized coefficients of a digital bandpass }\end{array}$ \\
\hline $\begin{array}{l}\text { Received Aug 29, } 2019 \\
\text { Revised Nov 7, } 2019 \\
\text { Accepted Mar 24, } 2020\end{array}$ & $\begin{array}{l}\text { filters with frequency sampling on its attenuation characteristic. It is shown } \\
\text { that only the coefficients, which depend on the frequency, when quantized, } \\
\text { significantly distort the shape of the attenuation characteristic. Such } \\
\text { coefficients in the article are called frequency coefficients. The magnitudes }\end{array}$ \\
\hline $\begin{array}{l}\text { Keyword: } \\
\text { digital filter } \\
\text { frequency coefficients } \\
\text { transfer function } \\
\text { attenuation characteristic } \\
\text { frequency sampling filters } \\
\text { digital comb of bandpass filters }\end{array}$ & $\begin{array}{l}\text { values at different values of the bit depth are analyzed. The attenuation } \\
\text { characteristics of the digital comb of bandpass filters based on frequency } \\
\text { sampling with calculated and quantized coefficients are analyzed. Practical } \\
\text { recommendations are offered to minimize the influence of frequency } \\
\text { coefficients quantization errors at the stage of solving the task of } \\
\text { approximation of digital bandpass filters with frequency sampling. The result } \\
\text { will be an optimal choice of the minimum number bit of the processors or } \\
\text { programmable logic integrated circuits (FPGA), to be used for the } \\
\text { implementation of digital bandpass filters with frequency sampling. }\end{array}$ \\
\hline
\end{tabular}

Copyright $\odot 2018$ Institute of Advanced Engineering and Science. All rights reserved.

\section{Corresponding Author:}

Varava Ivan Andriiovych,

Igor Sikorsky Kyiv Polytechnic Institute,

National Technical University of Ukraine,

Email: ivan.varava@ukr.net

\section{INTRODUCTION}

The study of applied implementation issues of multiband digital filtering systems, which have high computational efficiency, is of particular interest. It is advisable to implement such filter systems based on the Lagrange polynomial interpolation formula and, as a special case of their implementation, by the frequency sampling (FS) method.

Narrow-band filtering systems for broadband signals based on frequency sampling filters have high computational efficiency (minimum amount of calculations), guaranteed stability and linearity of phasefrequency characteristics [1]. If necessary, they can be easily rebuilt, both in software and in hardware. Frequency sampling filters (FSF) are effective FIR filters for implementing digital comb of bandpass filters (DCBF). With the help of FSF it is possible to process only a specified part of the operating frequency range. The "sliding" or "gating" mode of the analyzed frequency "windows" is easily realized. Filter systems based on FS can be useful in designing digital devices for spectral analysis, processing voice signals, quadrature phase shift keying and process control systems.

Developing an approximation technique, that is, finding a transfer function, when designing digital filters is a complicated scientific task. Research on the solution of the problem of filter approximation based on frequency sampling began in the 1970s [2, 3], was conducted in the 1990s and is ongoing to this day [48]. These are just some of the dozens of published works. The analysis of the mentioned works shows that at present there is no detailed elaborated and generally accepted method of solving the problem of approximation of digital filters by the method of frequency sampling. Successful synthesis of digital filters is 
not possible without a thorough study of the influence of the quantization of the transfer function coefficients on the frequency characteristics. The quantization effect is due to the use at the stage of implementation of devices with finite bit. Practical implementation of digital filters is carried out either by digital signal processors (DSP), or by programmable logic integrated circuits (FPGA, Field Field-Programmable Gate Array). At the same time, the coefficients of the filter calculated at the approximation step are quantized, i.e. their bit depth is limited. The choice of devices on which implementation is carried out, with high bit depth, leads to unnecessary hardware and cost and is not always possible. At present, much attention is paid to the development of new methods and approaches that reduce the hardware resources of FIR filters and increase the efficiency of hardware implementation on FPGAs [9-11].

The main difficulty that arises at the stage of implementation of filters with frequency sampling is the difference of coordinates of zeroes and poles (singular points) on the z-plane due to the quantization of the coefficients of the transfer function. The problem of finite bit depth and its associated negative effects is addressed in most fundamental work on digital signal processing, for example [12, 13]. One recent work [14] also addresses the issue of the coefficients' sensitivity and its minimization. In the mentioned works, the problem of limiting the bit depth is considered in general terms using the signal-to-noise ratio. The quantization effect of the transfer function coefficients and the input signal is considered as a noise source and the allowable signal-to-noise ratio is analyzed depending on the limitation of the bit depth and the filter order. Examples of determining the minimum allowable bit for some common types of digital filters with other transfer functions. However, the frequency dependence of quantization effects has not been analyzed. Circuit implementations with minimal quantization sensitivity and better signal-to-noise ratio are offered. For digital sampling of bandpass filters with frequency sampling, the problem of quantization has not been addressed. This determined the theme of this work. The research of the sensitivity of quantized coefficients is an urgent problem from a scientific and practical point of view. The article is aimed at research and concretization of scientific knowledge regarding the solution of applied problems of synthesis of filters with frequency sampling.

\section{PROPOSED RESEARCH METHOD / ALGORITHM}

Frequency sampling filter is a composite filter. It consists of a cascade-switched comb filter and parallel-connected digital resonators (a bank of resonators with real coefficients).

The modified transfer function of FSF has such a form [1]:

$$
H(z)=\left(1-t \times z^{-m}\right) \times \frac{1}{m} \times\left(1-z^{-2}\right) \times \sum_{k} \frac{A_{k} \times(-1)^{k}}{\left(1+b_{1} z^{-1}+b_{2} z^{-2}\right)},
$$

where: $\mathrm{z}^{-1}=\mathrm{e}^{-\mathrm{jx}}$;

$\mathrm{x}$ - normalized digital frequency ( $\mathrm{rad})$

$\mathrm{x}=\mathrm{w} \times \mathrm{T}=2 \times \pi \times \mathrm{f} / \mathrm{f}_{\mathrm{d}}$

$\mathrm{x} \in[0 \ldots \pi]$;

$\mathrm{f}_{\mathrm{d}}$ - sampling frequency;

$m$ - filter order;

$k$ - interpolation node number, $k=0 \ldots\left(\frac{m}{2}\right)$;

$b_{1 k}$ - resonator frequency coefficients that determine the resonance frequencies of digital resonators;

$A_{k}-$ weight coefficient;

$\frac{1}{m}-$ normalization coefficient;

$t$ - coefficient of the comb filter that is introduced to prevent the "multiplying by zero" state;

$b_{2}$ - phase coefficient, affects the linearity of the phase-frequency characteristic, which is introduced to ensure the stability of the filter and eliminate the state of "division into zero"; $b_{2}=1-2^{-d_{d}}$;

$d_{d}$ - the number of digits to express the fractional part of the coefficients;

$t=r^{+m}$.

The modified transfer function (1) differs from the previously considered transfer functions of FSF by the introduction of a second order comb filter link $\left(1-z^{-2}\right)$. Such a modification of the transfer function leads to frequency independence of the weighting coefficients $A_{k}$ due to the compensation of the constant nonlinear component. In this case $A_{k}=(-1)^{k} \times\left|H\left(i x_{k}\right)\right|$, so the weight factors is equal to the amplitudefrequency characteristic (AFC) value in the interpolation node. When solving the approximation problem, the values of the coefficients $A_{k}$ for the passband are chosen to be one. This greatly simplifies the task of 
implementation and the negative quantization effects of such coefficients are not apparent. The disadvantage here is the greater importance of the passband ripple. If the filters have sufficiently high requirements for the guaranteed damping in the stopband, then the so-called transition coefficients $A_{k}$ [1], which are determined by the frequency response in the transition band and not equal to one, should be introduced into the transfer function. The task of determining the values of these coefficients is central to solving the problem of FSF approximation. [1] provides tables with values of the transition coefficients for low pass filters. The number of transition coefficients can be from 1 to 3 . The numerical values of the transition coefficients are less than one and are calculated to within eight decimal places. This means that the accuracy of their implementation must be high and it is necessary to determine the influence of the quantization of the transition coefficients on the attenuation characteristic.

Practical experience in designing FSF demonstrates that the frequency coefficients of the resonator $b_{1 k}$ have the highest sensitivity in the transfer function (1). They are defined as the roots of a second order equation [13]:

The frequency coefficients $b_{1 k}$ of the resonators are defined as the roots of the second-order equation according to formula (1):

$$
b_{1 k}=-2 r \cos \left(x_{k}\right)
$$

where: $x_{k}-$ normalized digital frequency of interpolation nodes in the operating frequency range;

$x_{k}=k \times\left(\frac{2 \pi}{m}\right)$;

$k$ - interpolation node number;

$k=0 \ldots\left(\frac{m}{2}\right)$;

$r$ - pole distance;

$r=\sqrt{b_{2}}$;

$\left.b_{1 k} \in\right]-2 \ldots 2[$, because $r<1$.

The quantization errors of the frequency coefficients $b_{1 k}$ affect the coincidence accuracy of zeroes coordinates of the comb filter and the poles of the digital resonator on the $z$ - plane. When quantizing the frequency coefficients, the position of the pole frequencies shifts; as a result, the pole does not occur at the singular point of full zero compensation. As a result, there are local outliers of the attenuation characteristics $(\mathrm{AC})$ in the frequency range of the interpolation nodes. AC is defined as

$$
a(x)=-20 \times \lg (|H(z)|)[\mathrm{dB}]
$$

The following algorithm is suggested:

1. To analyze the influence of quantization of the transition coefficients $A_{k}$ on the attenuation characteristics of bandpass filters based on frequency sampling at different bit depth $d_{d}$.

2. To analyze the magnitude of the quantized frequency coefficients deviation from the calculated frequency coefficients of the FSF at different bit depth $-d_{d}$. Display the results of calculations on the graph.

3. To analyze the attenuation characteristic of a DCBF based on a frequency sample with calculated and quantized coefficients.

4. To analyze the filter attenuation characteristic on the FSF with quantized frequency coefficients with unit values of weight coefficients over the entire interval of the operating range of the normalized digital frequencies: $[0, \pi]$.

\section{RESULTS AND ANALYSIS}

3.1. Analysis of the influence of quantization of the transition coefficients $A_{k}$ on the attenuation characteristic

We will study using the transfer function of the form (1) for the order $m=118$, the values of the weighting coefficients $A_{24}=A_{25}=\ldots \ldots=A_{34}=1$ and the transition coefficients $A_{23}=A_{35}=0.385346$. These coefficients provide a bandwidth in the normalized frequencies from 1.325 to 1.764 and a delay band to 1.224 and from 1.863. The attenuation curve is shown in Fig. 1. The graphs show that the stopband attenuation is $\mathrm{a}_{\mathrm{g}}=41.1 \mathrm{~dB}$ and the passband ripple is $\Delta \mathrm{a}=0.65 \mathrm{~dB}$. 


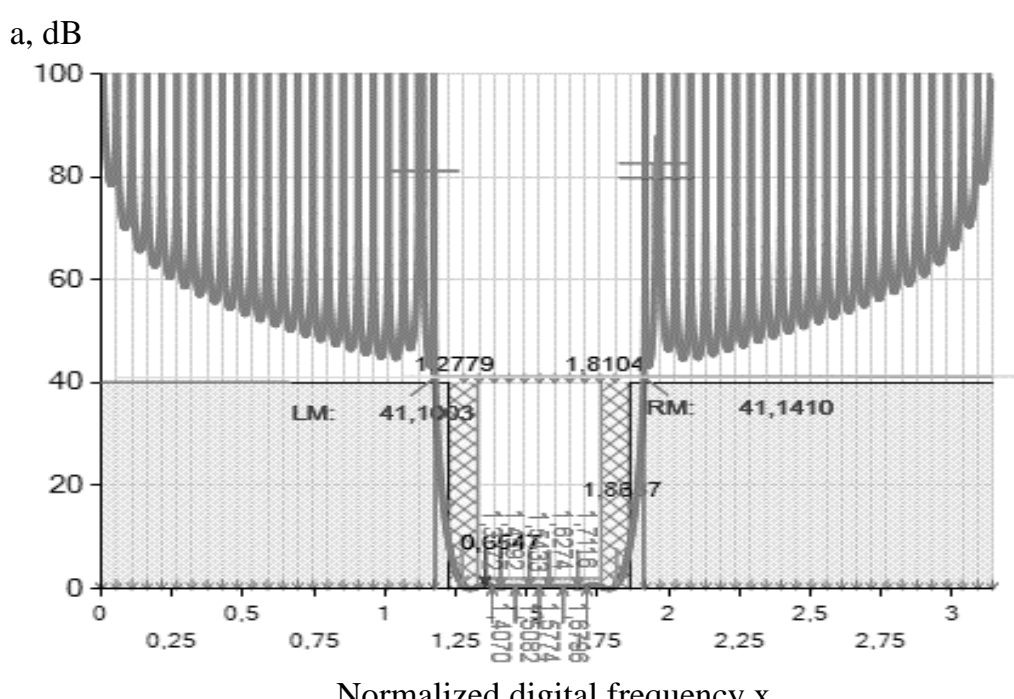

Figure 1. Frequency sampling filter attenuation characteristic in the range normalized frequencies from 0 to $\pi$

Table 1 shows the parameters of the attenuation characteristic with calculated and quantized transition coefficients.

Table 1. Results of the attenuation characteristic analysis of the bandpass filter based on frequency sampling with calculated and quantized transition coefficients

\begin{tabular}{cccc}
\hline Bit limits & $\mathrm{A}_{23}=\mathrm{A}_{35}$ & $\mathrm{a}_{\mathrm{g}}, \mathrm{dB}$ & $\Delta \mathrm{a}, \mathrm{dB}$ \\
\hline None & 0,385814 & 41,1 & 0,65 \\
$\mathrm{~d}=16$ & 0,385772 & 41,1 & 0,65 \\
$\mathrm{~d}=8$ & 0,375 & 42,1 & 0,75 \\
\hline
\end{tabular}

The analysis of the results shows that when the bit depth is limited to eight, there is a slight increase in attenuation in the stopbands and in the passband. That is, the quantization of the transition coefficients does not significantly affect the attenuation characteristic. The passband ripple varies within $0.1 \mathrm{~dB}$ and the stopband attenuation is within $1 \mathrm{~dB}$. It does not matter for practical implementation.

3.2. Analysis of the values of the quantized frequency coefficients deviation from the calculated frequency coefficients of the frequency sampling filters at different bit depth $-d_{d}$

We will analyze the deviations of the quantized frequency coefficients from the calculated frequency coefficients at different width $-d_{d}$ :

$$
\left|\Delta b_{1 k}\right|=\left|d b_{1 k}\right|-\left|b_{1 k}\right|
$$

where: $b_{1 k}$ - the calculated value of the frequency coefficient in accordance with (2);

$d b_{1 k}$ - quantized value of the frequency coefficient;

$\left|\Delta b_{1 k}\right|$ - the absolute value of the deviation of the quantized coefficient from the calculated.

The calculation of quantized frequency coefficients - $d b_{1 k}$ was performed by the rounding method at frequencies of zeroes in the range of normalized digital frequencies - $[0, \pi]$. The number of interpolation nodes $-k$ and the frequency of their location $-x_{k}$ depends on the order of the comb filter $-m$.

Fig. 2 shows the results of calculating the absolute values of the quantized frequency coefficients deviation from the calculated ones $-\left|\Delta b_{1 k}\right|$ in the form of a graph of functional dependence $-\left|\Delta b_{1 k}\left(x_{k}\right)\right|$.

The order of the comb filter is $m=120$ and the bit depth $-d_{d}=12$ (Fig. 2, curve 1), $d_{d}=18$ (Fig. 2, curve 2) is chosen arbitrarily to demonstrate the functional dependence $\left|\Delta b_{1 k}\left(x_{k}\right)\right|$. 


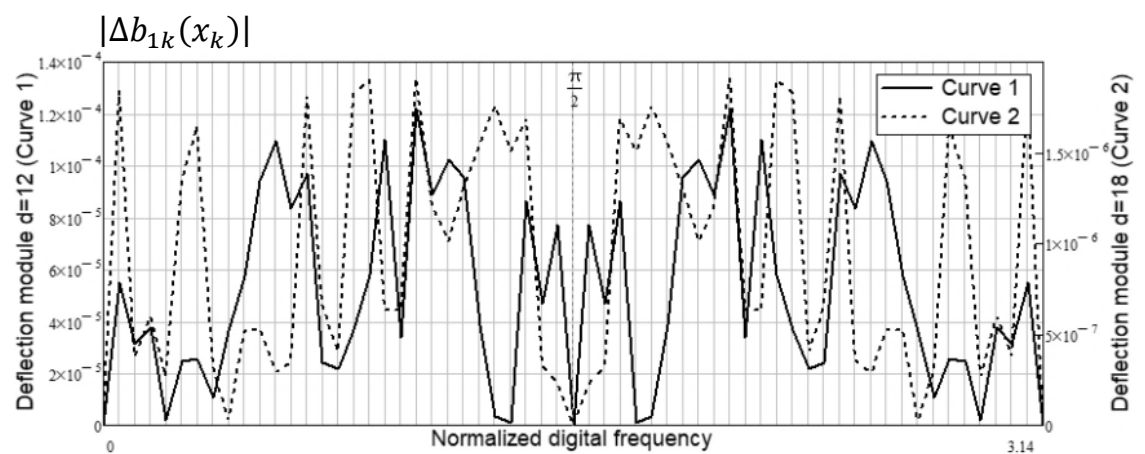

Figure 2. The magnitude of the modulus of quantized frequency coefficients deviation from the calculated interpolation nodes (Curve 1: $m=120, d_{d}=12$; curve 2: $m=120, d_{d}=18$ )

For $d_{d}=12$, one quantized level corresponds to $2^{-12} \approx 2.441 \cdot 10^{-4}$, and for $d_{d}=18$, one quantized level corresponds to $2^{-18} \approx 3.815 \cdot 10^{-6}$.

Analysis of the functional dependence $\left|\Delta b_{1 k}\left(x_{k}\right)\right|$ for different values of bit depth $d_{d}$-shows that it is equal to zero at the frequency $\pi / 2$ (for $m / 4$ even) and does not have a explicit nonlinearity in the entire frequency range from 0 to $\pi$. Graph $\left|\Delta b_{1 k}\left(x_{k}\right)\right|$ is mirror-symmetric about the frequency $\pi / 2$.

\subsection{Analysis of the attenuation characteristics of a digital comb of bandpass frequency sampling filters with calculated and quantized coefficients}

Consider the transfer function of DCBF based on FS. To save space in the text of the article we will limit ourselves to six comb outlets, which is enough for the analysis of the AC.

The transfer function of the DCBF on the basis of the FS on six outputs is:

$H_{l}(z)=\left(1-t \times z^{-m}\right) \times \frac{1}{m} \times\left(1-z^{-2}\right) \times\left[\begin{array}{l}\sum_{k 1=5}^{14} \frac{A_{k 1} \times(-1)^{k 1}}{\left(1+b_{1 k 1} z^{-1}+b_{2} z^{-2}\right)} \\ \sum_{k 2=13}^{22} \frac{A_{k 2} \times(-1)^{k 2}}{\left(1+b_{\left.1 k 2 z^{-1}+b_{2} z^{-2}\right)}\right.} \\ \sum_{k 3=21}^{30} \frac{A_{k 3} \times(-1)^{k 3}}{\left(1+b_{1 k 3^{2}} z^{-1}+b_{2} z^{-2}\right)} \\ \sum_{k 4=29}^{38} \frac{A_{k 4} \times(-1)^{k 4}}{\left(1+b_{1 k 4^{2}} z^{-1}+b_{2} z^{-2}\right)} \\ \sum_{k 5=37}^{46} \frac{A_{k 5} \times(-1)^{k 5}}{\left(1+b_{\left.1 k 5^{-1} z_{2} z^{-2}\right)}\right.} \\ \sum_{k 6=45}^{54} \frac{A_{k 6} \times(-1)^{k 6}}{\left(1+b_{1 k 6} z^{-1}+b_{2} z^{-2}\right)}\end{array} ;\right.$

where: $\quad l=1 \ldots 6 ; m=120 ; d_{d}=12$;

$l$ - number of DCBF outputs based on frequency sampling.

The weights are calculated by interpolation and optimized by the Chebyshev (minimax) proximity criterion. The values of the weighting coefficients for the outputs $l=1 \ldots 6$ in the groups $k_{1}, k_{2}, k_{3}, k_{4}, k_{5}, k_{6}$ (ten values each) are identical with each other (Table 2). The coefficients $b_{2}, b_{1 k}, t$ are calculated in accordance with (2).

Table 2. Table of weights of the DCBF with frequency sampling to six outputs in accordance with the transfer function (4)

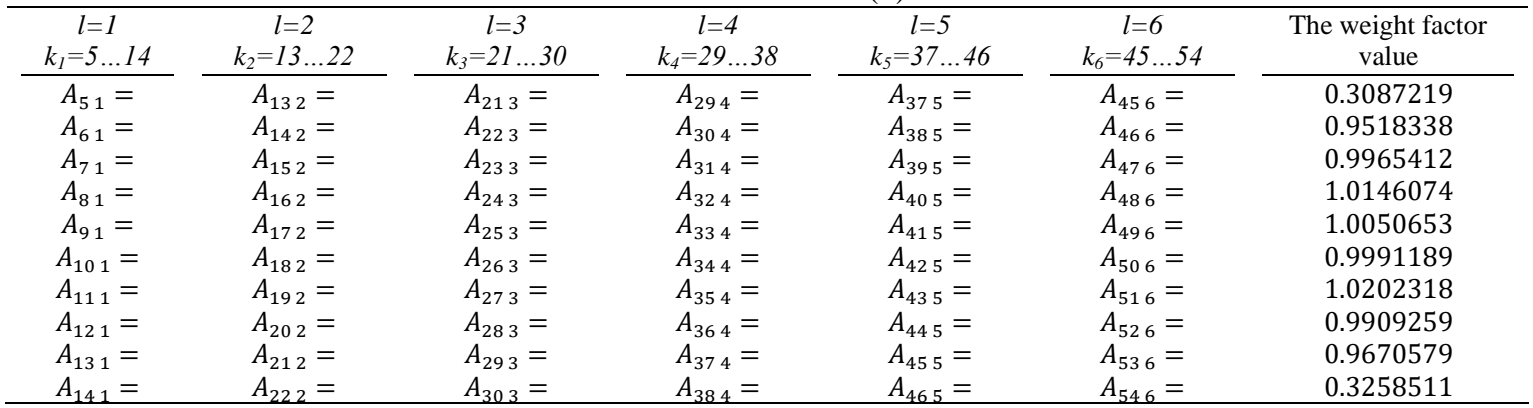


The attenuation characteristic (AC) of the DCBF in accordance with the transfer function (4), the calculated coefficients are $t, 1 / m, b_{1 k}, b_{2}$ and the weighting factors are $A_{k}$ in accordance with Table. 2 is shown in Fig.3.

a, dB

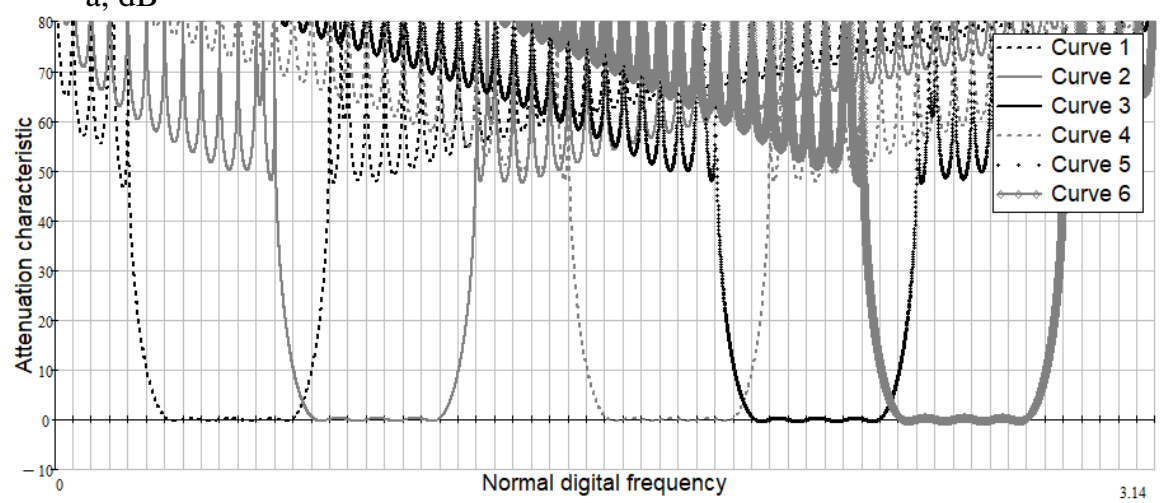

Figure 3. Attenuation characteristic of DCBF with calculated coefficients

Then the transfer function (4) coefficients were quantized by the rounding method. Distortions arose in bandwidths. For a detailed review of bandwidths in Fig. 4 shows AC according to the transfer function (4) and quantized coefficients. For ease of consideration, AC is depicted on a larger scale.

Research of the influence of transfer function (4) coefficients on the distortions of the AC showed that the outliers in Fig. 4 arise when quantizing the frequency coefficients $-b_{1 k}$. In this case, quantization was performed with the restriction of the discharge grid $d_{d}=12$.

For identical values of coefficients, except for frequency, for all six outputs, the amplitude of AC outliers in the bandwidth is different. The amplitude of outliers increases with distance from the normalized digital frequency $\pi / 2$ to the limits of the operating range of the normalized digital frequencies 0 and $\pi$.

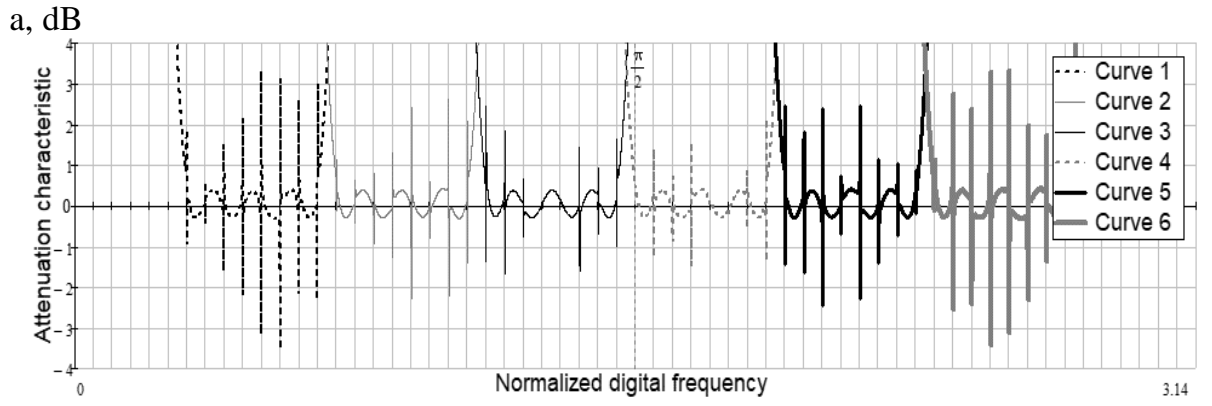

Figure 4. Attenuation characteristics of the bandpasses of the DCBF with quantized coefficients

3.4. Analysis of the attenuation characteristic of FCF with quantized frequency coefficients with unit weights throughout the entire range of the normalized digital frequencies ]0, $\pi[$

Let us consider the AC of FSF (1) under the condition $m=120, d_{d}=12$, and all the weighting factors are $A_{k}=1$ (for $k=1 \ldots 59$ ), i.e. with the exception of frequencies 0 and $\pi$ for the location of the poles. AC of FSF with calculated (not quantized) coefficients is shown in Fig. 3 (Curve 1).

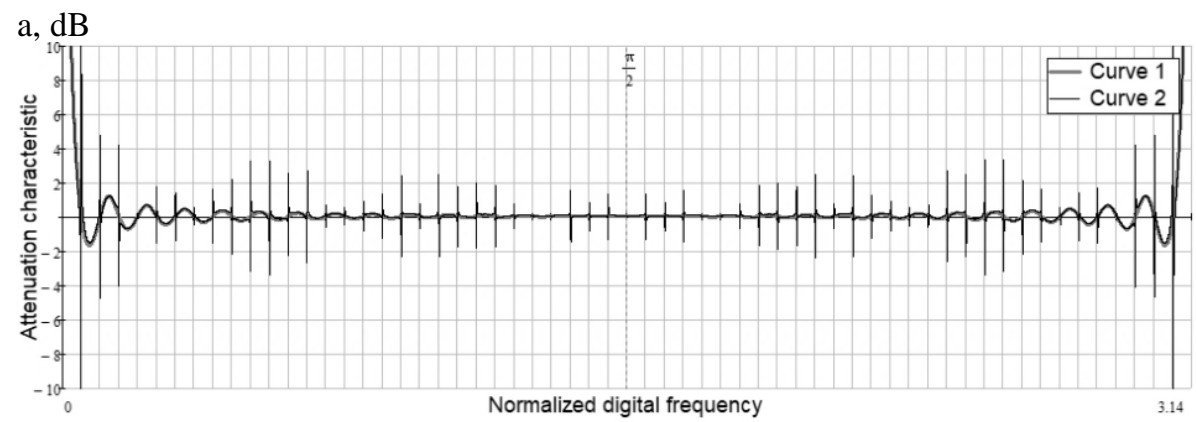

Figure 5. Attenuation characteristics of the FSF with calculated coefficients 
In Fig. 5 (Curve 1) the AC schedule runs at the interpolation nodes at the specified value - $0 \mathrm{~dB}$. There are no AC outliers in the interpolation nodes. In Fig. 5 (Curve 2) shows AC of the FSF (1) under the condition $m=120, d_{d}=12$, all weights $A_{k}=1$ (with $k=1 \ldots 59$ ) with quantized frequency coefficients. In Fig. 3 (Curve 2) it is clearly seen that in the working frequency range $(x \in[0 ; \pi])$ the AC of filter has spikes at the interpolation nodes. The amplitude of the outliers increases as the frequencies of the interpolation nodes tend to 0 and $\pi$.

Turning to Fig. 2 and Fig. 5 (Curve 2), it can be seen that the amplitude of the outliers of the AC in the working frequency range depends nonlinearly on the absolute value of the deviation of the quantized frequency coefficient - $\left|\Delta b_{1 k}\right|$ (Fig. 2). Nonlinearity is due to the functional dependence of the frequency coefficient on the digital frequency (2).

Let's perform an analysis of the functional dependence of the frequency factor $-b_{1 k}\left(x_{k}\right)$. When the order of the comb filter: $m=120$, the value of the frequency interval between the zeroes of the comb filter is equal to:

$$
2 \frac{\pi}{m} \approx 0.0524 \mathrm{rad}
$$

We calculate the difference between the frequency coefficients $b_{1 k}$ with $d_{d}=12$, at the interpolation nodes $k=0$ and 1 :

Calculate the quotient:

$$
\left|b_{10}\right|-\left|b_{11}\right| \approx 0.0027
$$

$$
\frac{\left(2 \frac{\pi}{m}\right)}{\left|b_{10}\right|-\left|b_{11}\right|} \approx 19.1 \text {. }
$$

Consequently, a change in the frequency coefficient from the interpolation node $k=0(k=0$ corresponds to frequency 0) to $k=1$ by the value of 0.0027 results in a frequency change of $0.0524 \mathrm{rad}$., which corresponds to a relative change 19.1 times. Taking into account the symmetry property of the frequency characteristics with respect to $\pi / 2$, as the frequencies tend to the interpolation node $k=60(k=60$ corresponds to the frequency $\pi$ ), the same dependence is observed.

We perform calculations between the interpolation nodes from $k=29$ to $k=30$ ( $k=30$ corresponds to the frequency $\pi / 2$ ):

Calculate the quotient:

$$
\left|b_{129}\right|-\left|b_{130}\right| \approx 0.1047 \text {. }
$$

$$
\frac{\left(2 \frac{\pi}{m}\right)}{\left|b_{129}\right|-\left|b_{130}\right|} \approx 0.5
$$

Consequently, a change in the frequency coefficient from the interpolation node $k=29$ to $k=30$ by the value of 0.1047 results in a frequency change of 0.0524 rad., which corresponds to a relative change of 0.5 times.

Based on the calculations performed, it can be concluded that the highest sensitivity of the quantized frequency coefficients is at frequencies tending to 0 and $\pi$. In these frequency areas of the interpolation nodes, a change in the $b_{1 k}$ coefficient by a small amount during quantization corresponds to a larger increment of the resonance frequency than at frequencies close to $\pi / 2$. As a result, the misregistration of the coordinates of zeroes and poles is greater, which entails a nonlinear increase in the amplitude of the outlier of the $\mathrm{AC}$ in the working frequency range.

With strict limitations of digit capacity, outlier of the AC can be compensated by reducing the pole distance - $r$. In this case, there is a "blurring" of the extremes of singular points, which lowers the sensitivity of the frequency coefficients. The coefficient $r$ is also called the attenuation coefficient. However, a decrease in the pole distance leads to a deterioration in the linearity of the phase-frequency response. Fig. 6 shows the AC of DCBF (4) with quantized coefficients, which is similar in the parameters of the AC DCBF (4) in Fig. 4. The only difference is that the pole distance is reduced: $r=\sqrt[2]{1-\left(2^{-12} \times 12\right)}$ (Fig. 5); $r=\sqrt[2]{1-\left(2^{-12}\right)}$ (Fig. 4).

$\mathrm{a}, \mathrm{dB}$

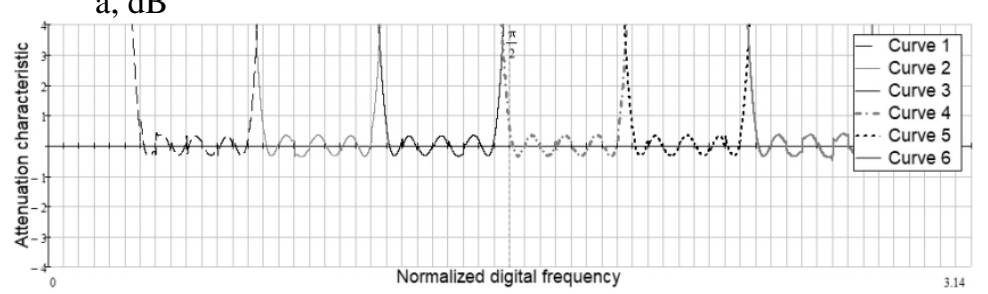

Figure 6. Attenuation characteristics of the bandpasses of the DCBF with quantized coefficients and a reduced pole distance 
It is known that the design (synthesis) of digital filters includes two main stages. The first is the approximation of specified requirements based on the use of discrete systems. The type of transfer function is selected, its order and the coefficients are calculated. The second is the realization of digital filters using arithmetic with limited accuracy. At this stage, the element base is selected and hardware-software realization of the digital filter is performed.

To achieve a more efficient design of digital filters, we suggest taking into account the quantization errors of the coefficients at the approximation stage.

The stage of accounting for the quantization errors of the transfer function coefficients (1) was successfully incorporated into the algorithm when developing a methodology for solving an approximate design problem for a DCBF based on FS. The results of the sensitivity survey of the frequency coefficients of the transfer function (4), which are presented in this article, were useful.

\section{CONCLUSION}

In the transfer function (1) of filters based on frequency sampling, only the frequency coefficients $b_{1 k}$ of the resonators are highly sensitive to quantization. The quantization of the weights coefficients $A_{k}$, regardless of their calculated values, has no effect on the filter attenuation characteristic. Analysis of the functional dependence of the quantized deviations from the calculated frequency coefficients of the FSF in the working frequency range $[0 \ldots \pi]$ at different bit widths shows that it is zero at the frequency $\pi / 2$ (at $m / 4$ paired) and has no pronounced nonlinearity. The graph of the functional dependence under consideration is symmetric with respect to the frequency $\pi / 2$.

An analysis of the attenuation characteristics of the digital comb of bandpass filters based on the frequency sampling showed that quantizing the frequency coefficients of the transfer function results in distortions of the AC in the form of outliers at the interpolation nodes. By the bandwidths of the DCBF, the outlier amplitude is different. The amplitude of the outlier has a functional dependence in accordance with the trigonometric function of double cosine of frequency.

The sensitivity of quantized frequency coefficients in the interpolation nodes is the highest at frequencies close to 0 and $\pi$, and the lowest at frequencies close to $\pi / 2$ of the working frequency range and has a symmetrical shape relative to the frequency $\pi / 2$. When designing digital combs of bandpass filters by the FS method, the non-uniformity of the AC in the passbands should be estimated by the worst result at frequencies close to 0 and $\pi$. With strict limitations of digit capacity, the outliers of the AC can be compensated by reducing the pole distance - r. However, a decrease in the pole distance entails a deterioration in the linearity of the phase-frequency characteristic of the FSF. In such a situation, it is important to find a balanced solution that should take into account the type of signals being processed in terms of sensitivity to phase nonlinearity. If the requirements for frequency characteristics at the selected bitness cannot be fulfilled, the bitness should be increased. The effect of quantization errors of the coefficients on the frequency characteristics of a digital filter should be taken into account at the approximation stage, which increases the design efficiency of DCBF using the frequency sampling method.

\section{REFERENCES}

[1] Lyons R. G. "Understanding Digital Signal Processing", 2nd Edition, published by Person Education, Inc., publishing as Prentice Hall Professional Reference Upper Saddle River, New Jersey, pp. 633-642, 2004.

[2] Rabiner L. R., Gold B. and McGonegal C. A, An approach to the approximation problem for nonrecursive digital filters, IEEE Trans. Audio Electroacoustics, Vol. 18, Issue 2, Jun 1970, pp. 83 - 106. - DOI: 10.1109/TAU.1970.1162092.

[3] J.H. McClellan and T.W. Parks, "A unified approach to the design of optimum FIR linear - phase digital filters", IEEE Trans. Circuit Theory, vol. CT - 20, pp. 697 - 701, Nov. 1973. - DOI: 10.1109/TCT.1973.1083764.

[4] Stubberud P.A., Leondes C.T., The design of frequency sampling filters by the method of Lagrange multipliers, IEEE Transactions on Circuits and Systems II: Analog and Digital Signal Processing, January 1993, Vol. 40, Issue 1, pp. 51 - 54. - DOI: 10.1109/82.215360.

[5] IEEE Transaction on Harris S.P., Ifeachor E.C., Automatic design of frequency sampling filters by hybrid genetic algorithm techniques Signal Processing, Vol. 46, Issue 12, December 1998, pp. 3304 - 3314. - DOI: $\underline{10.1109 / 78735305}$.

[6] Wan - Ping Huang, Li - Fang Zhou, Ji - Xin Qian, "FIR filter design: frequency sampling filters by Particle Swarm Optimization algorithm", Proceedings of the Third International Conference on Machine Learning and Cybernetics, pp. 2322 - 2327, Shanghai, 26 - 29 August 2004. - DOI: 10.1109/ICMLC.2004.1382187.

[7] R.Y. Belorutsky, I.S. Savinykh, Modified Technique of FIR Filter Design by the Frequency Sampling Method, IFOST - 2016: Information and Communication Technologies, pp. 259-262. DOI: 10.1109/IFOST.2016.7884100. 
[8] R.Y. Belorutsky, M.V. Oreshkina, I.S. Savinykh, The analytical approach for designing bandpass FIR filters by frequency sampling method, 2017 SIBIRCON, pp. 239 - 244. DOI: 10.1109/SIBIRCON.2017.8109879.

[9] Kumar C.V., Muthyala L., Chitra E. Design of a High Speed FIR Filter on FPGA by Using DA-OBC Algorithm // International Journal of Engineering Research and General Science. 2014. Vol. 2. Iss. 4. PP. 510-517.

[10] Badave S.M., Bhalchandra A.S. Multiplierless FIR Filter Implementation on FPGA // International Journal of Information and Electronics Engineering. 2012. Vol. 2. Iss. 2. PP. 185-188.

[11] Bhattacharjee S., Sil S., Chakrabarti A. Evaluation of Power Efficient FIR Filter for FPGA based DSP Applications // Procedia Technology. 2013. Vol. 10. PP. 856-865. DOI:10.1016/j.protcy.2013.12.431

[12] Rabiner L. R., Gold B. Theory And Application Of Digital Signal Processing, Prentice Hall, Englewood Cliffs, New Jersey, 1975, p.216.

[13] Oppenheim A.V., Schafer R.W. Discrete-Time Signal Processing, Prentice Hall, Englewood Cliffs, New Jersey, 1989, p.406.

[14] Takao Hinamoto, Wu-Sheng Lu, Digital Filter Design and Realization, River Publishers Series in Signal, Image and Speech Processing, 2017.- p.484 ISBN 8793519648, 9788793519640

IJEEI, Vol. 8, No. 1, March 2020 : $74-82$ 